\title{
Schizophreniform behavior in rats: Effects of L-dopa on various behavioral and physiological phenomena
}

\author{
KATHLEEN M. FELDKIRCHER, MARK T. FINNERAN, NICHOLAS E. NICOSIA, \\ HELEN M. MURPHY, and CYRILLA H. WIDEMAN \\ John Carroll University, Cleveland, Ohio
}

\begin{abstract}
The present study was conducted to investigate both the dopamine hypothesis of schizophrenia and the validity of using rats as animal models for schizophrenia. Male Sprague-Dawley rats were observed prior to and after intraperitoneal L-dopa injections. The following parameters were examined: (1) body weight, (2) wheel-running behavior, (3) open-field exploratory behavior, (4) grooming behavior, (5) body posture, and (6) urine ketone levels. Following intraperitoneal injections, the succeeding changes were noted: a decrease in mean gram body weight, a decrease in wheel-running behavior, a lack of exploratory behavior, an absence of grooming, a common occurrence of catatonic-like behavior in the home cage, and an increase in urine ketone levels. It is concluded that the rat is a good animal model for schizophrenia and that intraperitoneal injections of L-dopa do, in fact, produce symptoms suggestive of this disorder.
\end{abstract}

The chemical basis of schizophrenia comes from the observation that certain chemical abnormalities can produce schizophrenia or schizophrenia-like symptoms (Carlsson, 1978). The dopamine hypothesis of schizophrenia suggests a hyperactive dopamine system that may be related to either an increase in the sensitivity of the dopamine receptor or an increase of dopamine available in the synapse. It has been demonstrated that large doses of L-dopa, amphetamine, and methylphenidate can induce a schizophrenic state in normal subjects as well as cause patients diagnosed as schizophrenic to become more psychotic when given even low doses of the above chemicals (van Kammen, 1977). Stein and Wise (1971) utilized rats as an animal model to investigate the possible etiology of schizophrenia. They have postulated that there is a deficiency in the enzyme dopamine-beta-hydroxylase, which converts dopamine to norepinephrine. Consequently, there is a buildup of dopamine in the synapses that usually contain norepinephrine. Stein and Wise (1971) have further proposed that this excess of dopamine may be converted to 6-hydroxydopamine. This chemical, which destroys norepinephrine and dopamine synapses, causes progressive brain damage. There is also evidence that certain central noradrenergic pathways play a part in the reward mechanism and that these pathways degenerate in schizophrenia.

In order to cause a buildup of dopamine in the brains of rats, the chemical L-3,4-dihydroxyphenyl-

Requests for reprints should be sent to H. M. Murphy, Department of Psychology, John Carroll University, University Heights, Cleveland, $\mathrm{OH} 44118$. alanine (L-dopa) must be administered. L-dopa, which crosses the blood-brain barrier, is the precursor of dopamine. Once L-dopa reaches the brain, it is converted into dopamine (Brogden, Speight, \& Avery, 1971). Since the term "schizophrenia" cannot be appropriately applied to the animal model, the term "schizophreniform" is utilized in the present study. This state is characterized by a decrease in body weight, a decrease in wheel running, a decrease in open-field exploratory behavior, and a lack of grooming behavior. The purpose of the present study was to determine if a schizophreniform state could be induced by intraperitoneal (ip) injections of L-dopa in male Sprague-Dawley rats. Wheel-running activity and urine ketone levels were investigated prior to and after ip injections of L-dopa. The body weight of each animal was recorded on the first and last day of the series of ip L-dopa injections. Gross observations were also made on their behavior.

\section{METHOD}

\footnotetext{
Subjects

Nineteen male Sprague-Dawley albino rats, weighing between 240 and $400 \mathrm{~g}$ at the time of L-dopa injection, were utilized. The rats were individually housed in a room with a 12-h-light/12-hdark cycle. Room temperature was maintained between $72^{\circ}$ and $75^{\circ} \mathrm{F}$.

\section{Procedure}

The subjects were weighed on the first day of injection, and calculations were made to determine the dosage of L-dopa for each rat on the basis of $75 \mathrm{mg} / \mathrm{kg}$ of body weight. This dosage of $\mathrm{L}$ dopa was established by trial tests conducted in our laboratory. In a pilot study, it had been found that dosages above $200 \mathrm{mg} / \mathrm{kg}$ were lethal and dosages below $40 \mathrm{mg} / \mathrm{kg}$ had little behavioral effects on the animals. Thus, through trial tests, the optimal dose of
} 
$75 \mathrm{mg} / \mathrm{kg}$ of L-dopa was determined. Just prior to injection, the Ldopa was mixed in distilled water and acidified with several drops of concentrated hydrochloric acid until the L-dopa dissolved (Fink $\&$ Smith, 1979). All injections were performed between 8:00 and 8:30 a.m. Of the 19 rats used, 9 were assigned randomly to the wheel-running condition and the remaining 10 were assigned to the observatory condition. In all cases, each rat served as its own control. The body weight of each rat was recorded on the first day of ip L-dopa injection and the last day of ip L-dopa injection.

The nine rats assigned to the wheel-running condition were initially placed in the activity-wheel cage for a 5-day habituation period. The standard Wahmann activity wheel was incorporated in this part of the experiment. A $25 \times 15 \times 13 \mathrm{~cm}$ cage, which contained food and water, was attached to the wheel. A sliding door could separate the cage from the adjoining wheel. This door, however, was left open to allow the animal continuous access to the food and water. During the next 5 days, a baseline was recorded. Following the baseline period, a 5-day control period ensued. At this point, three of the nine animals received a $2-\mathrm{ml}$ ip injection of distilled water every $24 \mathrm{~h}$. The remaining six animals received no distilled water injection. Finally, for the last 5 days, all nine rats received an ip L-dopa injection every $24 \mathrm{~h}$. In each of the above conditions, the wheel-running activity was recorded over a $24-\mathrm{h}$ period.

Of the 10 rats assigned to the observatory condition, 5 were assigned randomly to an experimental group that had a 5-day baseline period, followed by a 5-day distilled ip water injection period, followed by a 5-day ip L-dopa injection period. The remaining 5 rats had a 5 -day baseline period, followed by a 5 -day ip L-dopa injection period. Water and L-dopa ip injections were administered every $24 \mathrm{~h}$. Gross observations were made $3 \mathrm{~h}$ after injection on the following areas: open-field exploratory behavior, grooming, and body posture. To test the animal's exploratory behavior, the rat was transferred from his home cage to the center of an open field $(167 \times 167 \mathrm{~cm}$ square area enclosed by a wooden barrier) and body movement was recorded. The floor of the field was divided by masking tape into $28-\mathrm{cm}$ square units, and the total distance the rat traveled in 5 min was tabulated. The animal's hygiene and posture while still in the home cage were observed. Particular attention was paid to the animal's general grooming habits, such as cleanliness. The body position that the animal assumed in the home cage was also noted.

Urinalysis was performed during both the wheel-running and observation conditions. Urine was collected every day between 11:00 and 11:30 a.m. and tested immediately using Labstix reagent strips. Area number 2 on the Labstix is sensitive to ketone levels ( $\mathrm{mg} / \mathrm{dL}$ ). According to the Ames Division of the Miles Laboratory, a positive result on the ketone portion of the Labstix indicates large amounts of leva-dopa metabolites.

\section{RESULTS}

The mean gram body weight was calculated for all 19 rats utilized in the present study (Figure 1). The mean gram body weight for the group prior to ip Ldopa injections was $339 \mathrm{~g}$. The mean gram body weight decreased to $305 \mathrm{~g}$ following 5 days of ip Ldopa injections. A $10.4 \%$ decrease in mean body weight was noted following the 5 days of L-dopa injection. Every animal, without exception, lost body weight.

The data for wheel-running behavior were analyzed using a $t$ test. A significant decrease in wheelrunning activity at the $p<.01$ level was found (Figure 2). During the control period the mean revolutions per $24 \mathrm{~h}$ increased from what they had been in the baseline period. However, a significant decrease

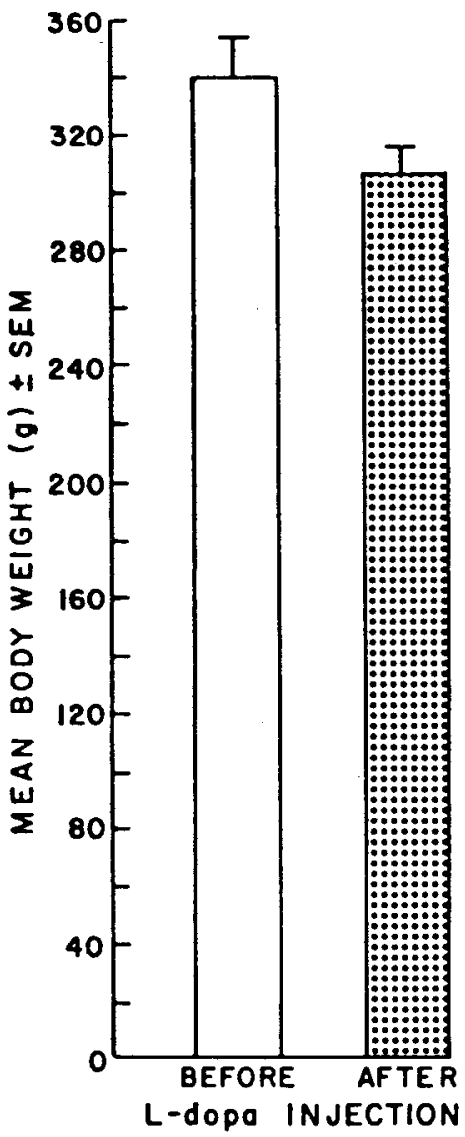

Figure 1. Mean gram body weight prior to and after ip L-dopa injections.

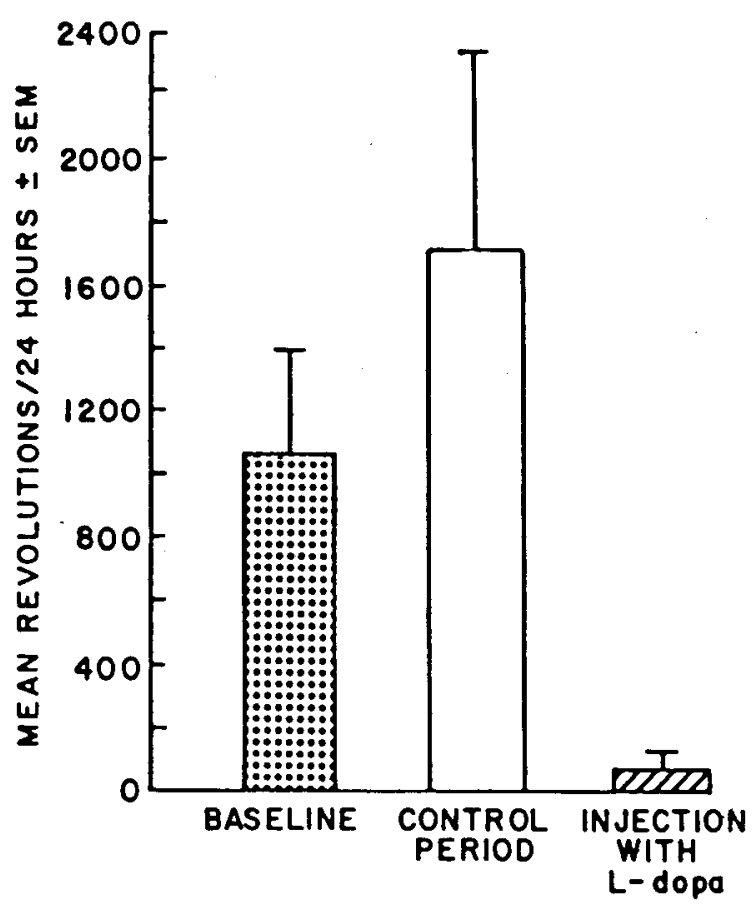

Figure 2. Mean wheel-running behavior during the baseline, control period, and ip L-dopa injection period. 
in mean revolutions was found during the L-dopa period: Three of the nine animals in the wheel-running condition received a $2-\mathrm{ml}$ ip distilled water injection every day during the control period. The remaining six animals received no distilled water ip injection during the control period. Since there were no significant differences between the two groups, the data were pooled, and thus the number of subjects in Figure 2 is nine.

It was found that the animals' open-field exploratory behavior diminished with the L-dopa injections. Prior to the L-dopa injection, the rats exhibited rearing and exploratory behavior, whereas during the $\mathrm{L}$ dopa injection period, they exhibited regressive behavior and little movement. The subjects appeared lethargic and would stare without blinking when the experimenter sounded a buzzer. The animals' openfield exploratory behavior when given the distilled water was not significantly different from that noted during the baseline period. The animals failed to groom themselves during the ip L-dopa injection period. Diarrhea was noted in all of the animals following the first ip L-dopa injection. By the end of the experimental session, fecal stains were found on the ventral side of the rats. The animals also exhibited catatonic-like behavior while in their home cages. Their bodies would remain rigid and in the same position for extended periods of time, and they were unresponsive to external stimuli (i.e., tapping on the front of the cage). A common position that a rat would assume was to lie on its back with its paws in the air.

There were increased ketone levels in the urine collected, as measured by the Labstix reagent strips. In the urine tested prior to ip L-dopa injections, a trace to moderate level of ketones was noted on the Labstix $(5-40 \mathrm{mg} / \mathrm{dL})$, thereby indicating large amounts of levadopa metabolites.

\section{DISCUSSION}

Intraperitoneal injections of L-dopa at a dosage of $75 \mathrm{mg} / \mathrm{kg}$ produces a schizophreniform state in male Sprague-Dawley rats. This schizophreniform state is manifested by a decrease in body weight, a decrease in wheel-running behavior, a decrease in open-field exploratory behavior, and a lack of grooming behavior. Furthermore, L-dopa induces a catatonic-like state in these rats. Unlike previous studies conducted with ip injection of L-dopa (Fink \& Smith, 1979; Schoenfeld \& Uretsky, 1973), the present study did not utilize an inhibitor of peripheral L-amino acid decarboxylase, which prevents the conversion of $\mathrm{L}$ dopa to dopamine. Thus, in animals in this study, dopamine levels could readily be increased.

The decrease in mean gram body weight that was observed following the last day of ip L-dopa injection is consistent with the adverse reaction noted in humans using levadopa. Anorexia and weight loss are possible side effects experienced by patients taking this drug (Brogden et al., 1971). It should be noted that the rats in the current study continued to increase their wheel-running activity during the control period, as compared with that observed in the baseline period. This phenomenon was also exhibited by the normal animals in Routtenberg and Kuznesof's (1967) study. It emphasizes the dramatic drop noted in wheel-running activity when L-dopa is introduced.

A decrease in exploratory behavior in an open field was observed in the present study. An increase in diarrhea and an absence of hygiene were noted in the animals. The rats also exhibited a catatonic-like state in the home cage while under the influence of $L$ dopa. The results suggest a lack of initiative. Crow (1980) contends that a flattened affect and a loss of drive are two of the negative symptoms seen in schizophrenic patients. Stein and Wise (1971) have postulated that affect flattening and anhedonia may result from a selective loss of neurons in the noradrenergic pathway.

The results of this study suggest a relationship between the administration of L-dopa and the onset of schizophreniform behavior. There are striking similarities between the behavior exhibited by the rats used in this study and the behavior observed in patients diagnosed as schizophrenic. The experimenters of this study concur with other investigators who believe that the rat can serve as an animal model to investigate schizophrenia.

\section{REFERENCES}

Brogden, R. N., Speight, T. W., \& Avery, G. S. (1971). Levodopa: A review of its pharmacological properties and therapeutic uses with particular reference to Parkinsonism. Drugs, 2, 262-400.

Carlsson, A. (1978). Antipsychotic drugs, neurotransmitters, and schizophrenia. American Journal of Psychiatry, 135, 164-173.

Crow, T. J. (1980). Molecular pathology of schizophrenia: More than one disease process? British Medical Journal, 280, 67-68.

Fink, J. S., \& SMiTH, G. P. (1979). L-dopa repairs deficits in locomotor and investigatory exploration produced by denervation of catecholamine terminal fields in the forebrain of rats. Journal of Comparative and Physiological Psychology, 93, 66-73.

Routrenberg, A., \& Kuznesof, A. (1967). Self-starvation of rats living in activity wheels on a restricted feeding schedule. Journal of Comparative and Physiological Psychology, 64, 414-421.

Schoenfeld, R. I., \& UREtsky, N. J. (1973). Enhancement by 6-hydroxydopamine of the effects of dopa upon the motor activity of rats. Journal of Pharmacology and Experimental Therapeutics, 186, 616-623.

SteIn, L., \& WISE, C. D. (1971). Possible etiology of schizophrenia: Progressive damage to the noradrenergic reward system by 6-hydroxydopamine. Science, 171, 1032-1036.

van Kammen, D. P. (1977). $y$-Aminobutyric acid (Gaba) and the dopamine hypothesis of schizophrenia. American Journal of Psychiatry, 134, 138-142.

(Manuscript received August 9, 1983; revision accepted for publication March 2, 1984.) 